\title{
Loss of peri-Wolffian duct stromal Frs2a expression in mice leads to abnormal ureteric bud induction and vesicoureteral reflux
}

\author{
Deepti Narla ${ }^{1}$, Stacey B. Slagle ${ }^{2}$, Caitlin M. Schaefer ${ }^{3}$, Daniel S. Bushnell ${ }^{3}$, Pawan Puri ${ }^{3}$ and Carlton M. Bates ${ }^{3}$
}

BACKGROUND: Fibroblast growth factor receptor 2 (Fgfr2) deletion from murine peri-Wolffian duct stroma (ST) results in aberrant ureteric bud induction, abnormal ureteral insertion into the bladder, and high rates of vesicoureteral reflux (VUR). It is unclear which receptor docking protein(s) is/are responsible for Fgfr2 actions in these tissues. We investigated whether the docking protein, fibroblast receptor substrate $2 a$ (Frs2a), had a role in peri-Wolffian duct ST similar to Fgfr2.

METHODS: We conditionally deleted Frs2a in peri-Wolffian duct ST with a Tbx18cre mouse line (Frs2 $a^{S T-/-}$ ). We assessed for ureteric induction defects and alterations in downstream targets mediating defects. We performed euthanized cystograms and assessed ureter-bladder junctions by three-dimensional (3D) reconstructions.

RESULTS: Embryonic day (E) 11.5 Frs2a $a^{S T-/-}$ embryos had many displaced ureteric bud induction sites when compared with controls. E11.0 Frs2a ${ }^{\text {ST-/- }}$ embryos had decreased Bmp4 expression and signaling, which can cause abnormal ureteric

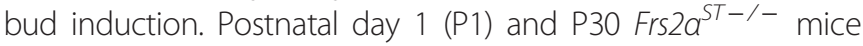
had higher VUR rates and grades vs. controls. Mutant refluxing ureters that inserted improperly into the bladder had shortened intravesicular tunnels (IVTs) when compared with controls

CONCLUSION: Frs $2 a^{S T-1-}$ embryos have aberrant ureteric induction sites, improper ureteral insertion, shortened intravesicular lengths, and VUR. Induction site defects appear secondary to reduced Bmp4 expression, similar to Fgfr2 mutants.

V esicoureteral reflux (VUR), retrograde flow of urine from bladder into kidneys, is present in $\sim 1 \%$ of children in North America (1). Although VUR appears to follow an autosomal-dominant inheritance pattern, no single-gene defect has yet been shown to cause the majority of reflux cases in patients. VUR can be associated with urinary tract infections, congenital renal and lower urinary tract malformations, and reflux nephropathy. Reflux nephropathy, characterized by severe renal scarring, is a common cause of end-stage renal disease in children and adults (2).
Aberrant ureteric bud induction has been identified as a cause of VUR (1). In early stages of renal development, the ureteric bud is induced to evaginate from the caudal mesonephric (Wolffian) duct by growth factors from the nearby metanephric mesenchyme (3). In the 1970s, Drs Mackie and Stephens hypothesized that improper ureteric bud induction site(s) from the Wolffian duct led to an abnormal position of ureteral insertion into the bladder, resulting in VUR (4). Additional studies have shown that reflux through abnormally positioned ureters is associated with shortened intravesicular ureteral tunnels and defects in bladder and ureter muscle (1). Under normal circumstances, the ureteric bud induction site is constrained to its proper position by repressive signaling from the stroma (ST) lying between the Wolffian duct and metanephric mesenchyme (5). One critical repressive factor from the ST is bone morphogenetic protein 4 (Bmp4), as even loss of one Bmp4 allele leads to ureteric induction defects in mice (5).

Fibroblast growth factor receptor (Fgfr) signaling appears to be critical for constraining ureteric bud induction and preventing reflux in mouse models. Although global deletion of Fgfr1 and/or Fgfr2 leads to early embryonic lethality, conditional knockout approaches have given insights into how the receptors regulate ureteric induction. Pax3cremediated deletion of Fgfr1 and Fgfr2 in both the metanephric mesenchyme and peri-mesonephric duct ST led to aberrant ureteric induction into a largely absent metanephric mesenchyme (6). Pax3cre-mediated deletion of Fgfr2 alone did not result in renal aplasia, but still often resulted in ureteric bud induction site abnormalities (too high or too low) with subsequent high rates of VUR $(7,8)$. Furthermore, Tbx18credriven loss of Fgfr2 in the ST but not the metanephric mesenchyme recapitulated the aberrant ureteral insertion and high-rate VUR, revealing that the receptor was acting through the ST alone to constrain the ureteric induction site (9). In both Pax3cre and Tbx18cre models, loss of Fgfr2 was associated with depressed Bmp4 expression $(9,10)$.

Fgfr signaling is mediated through docking adapter proteins, including Fgf receptor substrate $2 \alpha$ (Frs $2 \alpha$ ) among

\footnotetext{
${ }^{1}$ Akron Children's Hospital, Akron, Ohio; ${ }^{2}$ Department of Pediatrics, The Women's Hospital, Newburgh, Indiana; ${ }^{3}$ Division of Nephrology, Department of Pediatrics, University of Pittsburgh School of Medicine, Pittsburgh, Pennsylvania. Correspondence: Carlton M. Bates (batescm@upmc.edu)

Received 15 December 2016; accepted 21 June 2017; advance online publication 9 August 2017. doi:10.1038/pr.2017.175
} 
others. Frs $2 \alpha$ binds constitutively to the receptor juxtamembrane region and becomes phosphorylated upon receptor stimulation leading to the activation of Erk, Akt, and alternative forms of protein kinase C (11). While Frs $2 \alpha$ appears to drive most of the Fgfr signaling in metanephric mesenchyme (specifically nephron progenitors) (12), Frs $2 \alpha$ does not appear to interact with Fgfr2 in ureteric epithelium (13). Thus, the role of Frs $2 \alpha$ in mediating the effects of Fgfr2 signaling in peri-mesonephric duct ST is unclear. To answer this question, we used a Tbx18cre line to conditionally delete Frs $2 \alpha$ and examined the effects on ureteric bud induction, ureter insertion into the bladder, and rates of VUR.

\section{METHODS}

Mice

Mixed genetic background Tbx18cre; Frs $2 \alpha^{f l f l}$ mice (Frs $2 \alpha^{S T-/-}$ ) with deletion of Frs $2 \alpha$ in peri-Wolffian duct ST were produced by breeding Tbx18cre $e^{T g /+}$ mice (a gift from Dr Feng Chen (14)) that drive cre expression in the peri-Wolffian duct ST with Frs $2 \alpha^{f l / f l}$ mice (a gift from Dr Fen Wang). For embryonic studies, the day that vaginal plug was identified was deemed as E0.5. All experiments were carried out with approval of the University of Pittsburgh Institutional Animal Care and Use committee.

\section{Genotyping}

For PCR genotyping, DNA was extracted from tail clippings or embryonic tissues as previously described (9). Table 1 lists the primers and fragment sizes for various genes.

\section{Cystograms}

To assess for reflux, euthanized cystograms were performed in P1 and P30 Frs $2 \alpha^{S T-1-}$ and littermate control pups as previously described (9). Briefly, the mice were killed and abdominal walls were reflected to expose the bladder. 30 -gauge and 25-gauge needles were inserted into the bladders of $\mathrm{P} 1$ and $\mathrm{P} 30$ mice, respectively. Then, the bladders were gravity-filled with $1 \%$ methylene blue dye in a $50 \mathrm{ml}$ syringe raised $30 \mathrm{~cm}$ at $5-\mathrm{s}$ intervals starting at a height of $0 \mathrm{~cm}$ to a maximum height of $150 \mathrm{~cm}$. Refluxing dye from the bladder toward the kidney (if any) was graded on a I-V scale according to the standard international classification (15). At the completion of the cystogram, all kidneys were photographed and kidney long axis was assessed using Image J software (National Institutes of Health, Bethesda, MD).

\section{Post-Cystogram Three-Dimensional Reconstructions}

Following P1 cystograms, lower bodies from control and Frs $2 \alpha^{S T-/-}$ mice were fixed in $4 \%$ paraformaldehyde/phosphate-buffered saline (PFA/PBS) overnight at $4{ }^{\circ} \mathrm{C}$, dehydrated, and paraffin-embedded. The tissues were serially sectioned at $10 \mu \mathrm{m}$ from the junction of the ureters and bladder to the bladder neck, and then stained with hematoxylin and eosin $(\mathrm{H} \& \mathrm{E})$. The tissues were reconstructed using
Stereo Investigator, insertion angles calculated, and IVT lengths determined using Neurolucida (MBF Biosciences, Williston, VT) as previously described (9).

\section{P30 Bladder Histology and Immunofluorescence}

Paraffin-embedded tissues $(6 \mu \mathrm{m})$ were stained with H\&E. Sections were also immunostained as previously described (13) using primary antibodies against $\alpha$ SMA (1:250; Sigma Aldrich, St Louis, MO), followed by the use of secondary antibodies, donkey anti-mouse 594 Rabbit (1:500; Molecular Probes, Grand Island, NY), and DAPI $(1: 10,000)$.

\section{In Situ Hybridization}

Whole-mount in situ hybridization (WISH) was performed on E11.5 control and Frs $2 \alpha^{S T-1-}$ tissues as previously described (9). Briefly, DNA fragment templates for Bmp4 (Gene Accession number: NM_007554), Frs2 $\alpha$ (NM_177798), and Ret (NM_001080780) were ligated into plasmids using pGEM-T Easy Vector system (Promega, Madison, WI). The plasmids were then introduced into competent cells (Promega), after which the cells were cultured on Blue/White Select Ampicillin-treated agarose plates overnight at $37^{\circ} \mathrm{C}$. White colonies were selected and underwent DNA isolation using QIAprep Spin Miniprep Kit (Qiagen, Hilden, Germany). Following isolation, the plasmid samples were digested using restriction enzyme (EcoRI), sequenced, and linearized using PCR primers designed for M13 sites. Linearized samples were used to generate digoxigenin UTP-labeled antisense RNA probes using DIG RNA Labeling kit as per the manufacturer's instructions (Roche, Basel, Switzerland).

For in situ hybridization, 4\% PFA/PBS-fixed tissues were washed in PBS-Tween (PBST) and treated with $6 \% \mathrm{H}_{2} \mathrm{O}_{2}$ in PBST, followed by $10 \mu \mathrm{g} / \mathrm{ml}$ Proteinase $\mathrm{K}$ in PBST. The samples were re-fixed in $4 \%$ PFA/PBS, washed in PBST, and incubated overnight at $58^{\circ} \mathrm{C}$ in a hybridization solution containing DIG RNA probe (1:200). On the following day, the samples were washed blocked in 1\% Blocking solution (Roche) in Maleic acid buffer with tween (MABT), and were incubated overnight at $4{ }^{\circ} \mathrm{C}$ with 1:2,000 anti-DIG antibody (Roche). The samples were subsequently washed in MABT, and developed in $\mathrm{BM}$ purple $+0.1 \%$ Tween at $4{ }^{\circ} \mathrm{C}$ with color change observed every $8 \mathrm{~h}$.

\section{Western Blot Analysis}

Protein samples $(30 \mu \mathrm{g}$ each) from six whole E11.5 control and Frs $2 \alpha^{S T-1-}$ urogenital ridges were resolved in $8 \%$ SDS-Tris gels (Cat\#161-0732, Bio-Rad, Hercules, CA). The proteins were electrotransferred to nitrocellulose membranes (Cat\#162-0115, Bio-Rad), blocked with $5 \%$ non-fat dry milk in TBST, and were incubated overnight at $4{ }^{\circ} \mathrm{C}$ with primary antibodies (pSmad 1/5/8 1:1,000; Cat\#9511, Cell Signaling and $\beta$-Actin: 1:1,000, Cat\#8457, Cell Signaling, Beverly, MA). The membranes were washed with TBST, and probed with HRP-conjugated secondary antibodies (1:2,000; Anti-Rabbit HRP Cat\#7076, Cell Signaling). Bound antibodies were visualized using the Amersham ECL Prime Western Blotting Detection kit (Cat\#RPN2232GE, Lifesciences, Pittsburgh, PA) according to the manufacturer's instructions. The blots were imaged

Table 1. Primers used for genotyping

\begin{tabular}{lll}
\hline Gene & Primer pairs & Size (bp) \\
\hline Tbx 18cre & 5'-CCA TCC AAC AGC ACC TGG GCC AGC TCA ACA-3' & 500 (wt) \\
Frs2a & 5'-CCA CCA TCG GTG CGG GAG ATG TCC TTC ACT-3' & 250 (mt) \\
& 5'-GAG TGT GCT GTG ATT GGA AGG CAG-3' & 224 (wt) \\
CAG & $5^{\prime}-$ GGC ACG AGT GTC TGC AGA CAC ATG-3' & 319 (mt) \\
& 5'-AAG GGA GCT GCA GTG GAG TA-3' & 297 (wt) \\
& 5'-CCG AAA ATC TGT GGG AAG TC-3' & \\
& 5'-GGC ATT AAA GCA GCG TAT CC-3' & 196 (CAG-positive) \\
\hline
\end{tabular}


using a Fujifilm LAS-3000 (Fujifilm Medical Systems, Stamford, CT) and densitometry analysis was performed using Image J Software.

\section{Common Nephric Duct Length}

To determine the length of the common nephric duct (CND; the caudal portion of the Wolffian duct between the ureteric bud and the urogenital sinus; CND), six E11.5 Frs $2 \alpha^{S T-/-}$ and control embryos were immersion-fixed overnight in 4\% PFA/PBS and embedded in paraffin. Tissues along the Wolffian duct were serially sectioned at $4 \mu \mathrm{m}$ from the cloaca to the base of the ureteric bud and stained with $\mathrm{H} \& \mathrm{E}$. Three-dimensional (3D) images were rendered using Stereo Investigator and CND lengths (from the base of the ureteric bud to the cloaca) were determined using Neurolucida software (MBF Biosciences, Williston, VT) as previously described (9). Data are represented as mean \pm standard deviation.

\section{CND Cell Proliferation and Apoptosis}

To examine proliferation and apoptosis in the CND, E12.5 Frs $2 \alpha^{S T-1-}$ and littermate control embryos were fixed in $4 \%$ PFA/PBS, embedded in paraffin, and serially sectioned at $5 \mu \mathrm{m}$ along the CND. For proliferation analysis, the sections were labeled with antibodies against phospho-histone $\mathrm{H} 3$ (pH3: 1:200; Sigma Aldrich) and E-Cadherin (Ecad: 1:250; Sigma Aldrich) to visualize proliferating cells and the CND epithelium, respectively. Phospho-histone H3 was detected with goat anti-rabbit Alex Fluor 594 and E-Cadherin was visualized with donkey anti-rat Alex Fluor 488 (1:500; Molecular Probes). To identify apoptotic cells, terminal deoxynucleotidyl transferase-mediated dUTP Nick End Labeling (TUNEL) was performed using ApopTag Fluorescein Direct in situ Apoptosis Detection Kit (Cat\#S7160 EMD Millipore, Darmstadt, Germany) according to the manufacturer's instructions. Quantification of apoptosis and proliferation along the CND (number of cells per $100 \mathrm{CND}$ cells) were determined ( $n=3$ per genotype) using Image J Software.

\section{Statistics}

One-way analysis of variance followed by Bonferroni's post hoc analysis, Student's T-tests, or Fisher's exact test analyses were conducted where appropriate, using GraphPad Prism 5 (GraphPad Software, La Jolla, CA).

\section{RESULTS}

The Tbx18cre Line Efficiently Deletes Frs2a Expression in PeriWolffian Duct ST

To determine the role of Frs $2 \alpha$ in peri-Wolffian duct ST, we generated Tbx18cre; Frs $2 \alpha^{f l f l}$ mice (Frs $2 \alpha^{S T-/-}$ ) with deletion of Frs $2 \alpha$ in peri-Wolffian duct ST. As shown in (ref. 9), breeding a CAG reporter mouse to the Tbx18cre line reveals significant cre recombination throughout the Wolffian duct ST at E10.5. Before examining the defects in these mice, we determined the efficiency of Frs $2 \alpha$ deletion in Frs $2 \alpha^{S T-/-}$ mice, by performing WISH in control and Tbx18cre; Frs $2 \alpha^{F l / F l}$ E10.5 embryos. WISH illustrated a significant reduction in Frs $2 \alpha$ mRNA expression in mutant peri-Wolffian duct ST at E10.5 when compared with age-matched controls (Figure 1a,b).

\section{Frs $2 a^{\mathrm{ST}-/-}$ Embryos have Abnormal Ureteric Bud Induction}

Given that Fgfrs utilize different docking proteins to transmit downstream signaling, we determined whether Tbx18cremediated deletion of Frs $2 \alpha$ in the peri-Wolffian duct ST affected ureteric induction similar to Tbx18cre; Fgfr $2^{f l f l}$ mutants $(8,9)$. Thus, we measured CND length (Wolffian
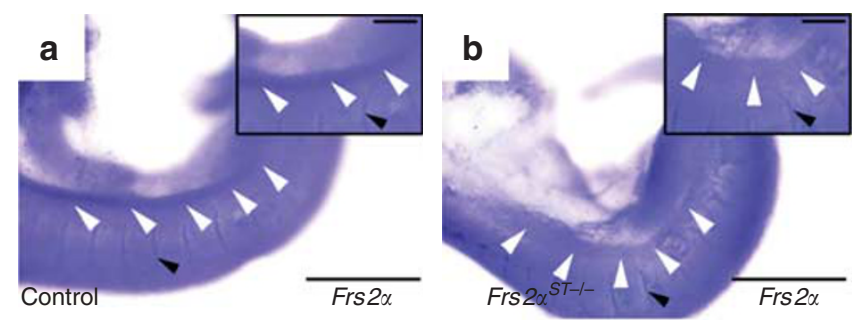

Figure 1. Frs $2 a$ expression is significantly reduced in E10.5 Frs $2 a^{S T-1-}$ peri-Wolffian duct stromal cells. (a,b) Representative whole-mount in situ hybridization shows dramatically reduced peri-Wolffian duct Frs $2 a$ mRNA expression (purple, white arrowheads) in Frs $2 a^{S T-/-}$ embryos (b) at E10.5 when compared with age-matched controls (a). Importantly, intersomite Frs2a mRNA expression (black arrowheads) is comparable between both genotypes. (Insets in $\mathbf{a}$ and $\mathbf{b}$ represent higher magnifications of the peri-Wolffian duct regions in the embryos. (a,b) Bar $=500 \mu \mathrm{m}, \mathrm{Bar}=150 \mu \mathrm{m}$ (inset).

duct segment between the cloaca to the base of the ureteric bud) in E11.5 control and Frs $2 \alpha^{S T-/-}$ embryos. 3D reconstructions revealed similar CND lengths on each side of controls, but frequently divergent lengths on each side of the mutants (Figure 2a,b). Although mean CND lengths were comparable between both genotypes (control $\left.195.84 \pm 9.90 \mu \mathrm{m} ; \quad F r s 2 \alpha^{S T-/-}: 223.96 \pm 57.98 \mu \mathrm{m}, P=0.8\right)$, Frs $2 \alpha^{S T-1-}$ embryos had a wide distribution of CND lengths, whereas controls had CND lengths tightly clustered around their mean (Figure 2c). Following analysis of five paired sets of control and Frs $2 \alpha^{S T-/-}$ embryos, we observed 70\% (7/10) of control CND lengths were within $1 \mathrm{SD}$ of the mean control CND length mean value, whereas, only $10 \%(1 / 10)$ of Frs $2 \alpha^{S T-I-}$ CND lengths were within $1 \mathrm{SD}$ of the mean control CND length (Figure 2c). As suggested by the 3D reconstruction images (Figure 1a,b), intra-embryo variation in CND length is also significantly greater in $\operatorname{Frs} 2 \alpha^{S T-1-}$ embryos when compared with that in controls (Figure 2d). Thus, similar to Tbx18cre; Fgfr $2 f^{l / f l}$ mice, Tbx18cre; Frs $2 \alpha^{f l f f l}$ embryos have significant ureteric bud induction defects characterized by a virtual randomization of inductions sites along the Wolffian duct.

\section{CND Proliferation and Apoptosis is Similar in Both Genotypes} We then assessed whether intrinsic defects in the CND (i.e., aberrant proliferation and/or apoptosis) could be responsible for the induction defects and altered CND lengths in the Frs $2 \alpha^{S T-/-}$ mutants. Phospho-histone H3 staining of control and Frs $2 \alpha^{S T-/-}$ CND indicates comparable mean numbers of proliferating cells in both genotypes (control: $3.77 \pm 0.50$ $\times 10^{-2}$ cells per 100 CND cells; Frs $2 \alpha^{S T-/-}: 4.24 \pm 1.11$ $\times 10^{-2}$ cells per $100 \mathrm{CND}$ cells, $P=0.54$ ) (Supplementary Figure S1 online). In addition, TUNEL staining of control and Frs $2 \alpha^{S T-1-}$ CNDs illustrated similar levels of apoptosis (control: $26.06 \pm 16.73 \times 10^{-2}$ cells per $100 \mathrm{CND}$ cells; Frs $2 \alpha^{S T-/-}: 32.75 \pm 8.11 \times 10^{-2}$ cells per $100 \mathrm{CND}$ cells. $P=0.57$ ) in both genotypes (Supplementary Figure S1). Thus, changes in CND cell proliferation or apoptosis do not 

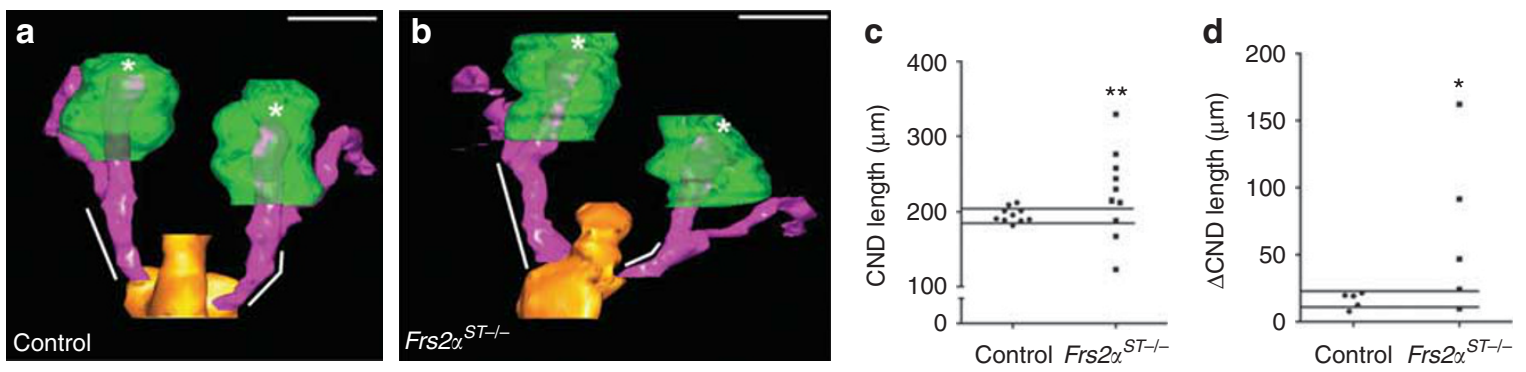

Figure 2. E11.5 Frs2 $a^{S T-/-}$ embryos exhibit aberrant ureteric bud induction. (a,b) 3D reconstructions of E11.5 control and Frs2a $a^{S T-/-}$ urogenital tissues reveal common nephric duct lengths (white lines) of greater variability in Frs $2 a^{S T-/-}$ embryos (b) vs. controls (a). (c) Graph illustrating that most control CND lengths are within 1 SD of the control mean length (lines), whereas Frs $2 a^{S T-/-}$ CND lengths are frequently $>1$ SD on either side of the control mean length. (d) Graph illustrating that control intra-embryonic CND lengths are almost all within 1 SD of the control mean length (lines), whereas almost all Frs2 $a^{S T-1-}$ intra-embryonic CND lengths are $>1$ SD on either side of the control mean length ( $n=5$ embryos per genotype). Fisher's exact test: ${ }^{*} P<0.05,{ }^{* *} P<0.01$. Green-metanephric mesenchyme; orange-Cloaca; pink-Wolffian duct and ureteric bud; asterisks—ureteric bud tips. Bar $=200 \mu \mathrm{m}$. CND, common nephric duct.
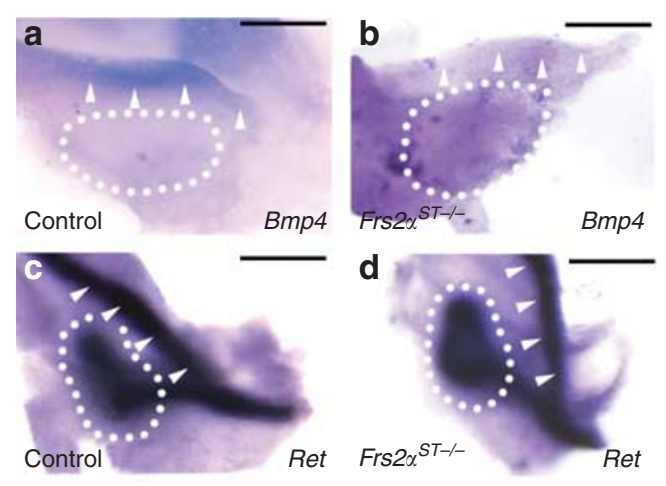

e
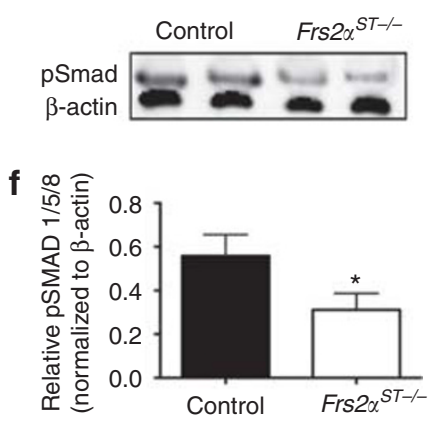

Figure 3. Bmp4 expression and canonical signaling is reduced in E11.5 Frs $2 a^{S T-/-}$ peri-Wolffian duct stroma. (a,b) Representative whole-mount in situ hybridization in urogenital ridges reveals that compared to the control (a), Bmp4 expression is virtually absent adjacent to the Wolffian duct (arrowheads) in Frs2 $a^{S T-1-}$ embryos (b, arrowheads). (c,d) In contrast to Bmp4, in situ hybridization reveals similar Ret expression in Wolffian ducts (arrowheads) of the control (c) and mutant (d). (e) Representative western blot analysis for pSmad 1/5/8 (pSmad) expression in control and Frs $2 a^{S T-/-}$ urogenital ridges suggests reduced canonical Bmp signaling in Frs $2 a^{S T-/-}$ tissues. (f) Ratios of pSMAD $1 / 5 / 8$ and $\beta$-actin loading control bands quantified by densitometry confirms a significant reduction in canonical Bmp4 signaling. Dotted lines encircle the metanephros $(n=3)$. Values $=$ mean + SD. Student's test $=* P<0.05$. Bar $=200 \mu \mathrm{m}$.

lead to changes in CND length observed in Frs $2 \alpha^{S T-/-}$ embryos; this suggests that defects in signals from the adjacent peri-Wolffian duct ST likely drive the induction defects.

\section{Frs $2 a^{\text {ST-I- }}$ have Reduced Bmp4 Expression in Peri-Wolffian \\ Duct ST}

We then determined whether the aberrant ureteric bud induction observed in $\operatorname{Frs} 2 \alpha^{S T-1-}$ mice was associated with a decrease in Bmp4 expression, similar to Fgfr2 mutants. WISH on the urogenital ridges revealed significantly reduced $B m p 4$ mRNA expression in Frs $2 \alpha^{S T-/-}$ peri-Wolffian ST (Figure 3a,b). Furthermore, expression of Ret, a marker of Wolffian duct and ureteric epithelium, appeared unaltered in mutants vs. controls, making it unlikely that alterations in Ret expression were responsible for the induction defects (Figure 3c,d). Western blot analysis revealed about a $43 \%$ decrease in phosphorylated Smad 1/5/8 expression in E11.5 $F r s 2 \alpha^{S T-/-}$ urogenital ridges vs. controls (Figure 3e,f), strongly suggesting that the loss of mutant $B m p 4$ mRNA led to a decrease in canonical Bmp4 signaling. Thus the loss of Frs2 in peri-Wolffian duct ST appears to cause reduced Bmp4 expression and canonical Bmp4 signaling, which may explain the aberrant ureteric bud induction in Frs $2 \alpha^{S T-1-}$ embryos.

\section{Frs $2 a^{\text {ST-I- }}$ Mice have High Rates of VUR at Birth}

To determine whether the ureteric induction defects in $\operatorname{Frs} 2 \alpha^{S T-/-}$ mice correlate with increased susceptibility for VUR, we performed cystograms in P1 control and Frs $2 \alpha^{S T-/-}$ dissected urinary tracts (Figure 4). P1 Frs $2 \alpha^{S T-/-}$ mice exhibit significantly higher rates of VUR (48.3\%) when compared with the VUR rates in age-matched controls $(6.9 \% ; P<0.001)$ (Table 2). The higher rates of reflux held true when comparing female or male mutants with their gender-matched controls (Table 2). Although most reflux (mutant or control) was unilateral, all of the P1 controls had low-grade (I-II) reflux, whereas $31 \%$ of refluxing mutants had high-grade (III-IV) VUR (Table 2). Thus, P1 mutants have significantly higher 


\section{Articles | Narla et al.}

rates and grades of reflux when compared with those in their littermate controls.

Location and length of ureteral insertion into the bladder are key components determining susceptibility for VUR and aberrant ureteric induction is associated with abnormal ureter insertion into the bladder. To investigate ureteral insertion, we reconstructed ureter-bladder junctions in control, nonrefluxing $\operatorname{Frs} 2 \alpha^{S T-/-}$, and refluxing (unilateral) $\operatorname{Fr} 2 \alpha^{S T-/-}$ mice (Figure 5). In control and non-refluxing mutants, the ureters appeared to insert at a similar location on the bladder, whereas the mutants with unilateral reflux had dissimilar insertion sites; the ureters with reflux inserted either high and lateral or low and medial to the non-refluxing ureter (Figure 5a,b and not shown). Triangles generated from the (internal or external) insertion sites of the ureters and the bladder neck generally were close to isosceles triangles in controls and nonrefluxing mutants, whereas they were distorted in mutants with unilateral VUR (Figure $\mathbf{5 c , d}$ and not shown). Measurements of either the external or internal angles at the site of ureter insertion revealed a much larger difference in mutants with unilateral reflux than either in control or in non-refluxing $F r s 2 \alpha^{S T-/-}$ mice (Figure 5e,f). Moreover, mean IVT lengths (i.e., the distance between the external and internal ureter insertion points) of refluxing $\operatorname{Frs} 2 \alpha^{S T-/-}$ ureters were significantly shorter than control and nonrefluxing $F r s 2 \alpha^{S T-/-}$ mean IVT lengths (Figure 5g). Individual H\&E-stained sections used for 3D reconstructions show that the IVTs for the refluxing mutant ureters have a linear, straight shape, whereas the nonrefluxing mutant ureters and control ureters appear to have
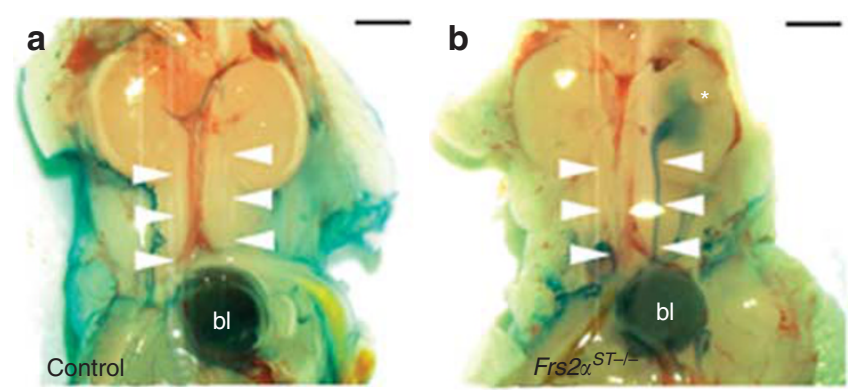

Figure 4. Cystograms in $\mathrm{P} 1 \mathrm{Frs} 2 \mathrm{a}^{\mathrm{ST-1}-}$ and control mice. (a) Representative image of a control cystogram revealing methylene blue dye in the bladder (bl), no dye present in the ureters (arrowheads). (b) Image of a Frs $2 a^{S T-/-}$ cystogram illustrating dye present in the bladder that tracked up the left ureter into the left renal pelvis $\left(^{*}\right)$. Bar $=500 \mu \mathrm{m}$. a more curvilinear trajectory (consistent with smaller tunnel lengths within refluxing mutant ureters; Supplementary Figure S2). These data indicate that $F r s 2 \alpha^{S T-/-}$ mice have an increased susceptibility and severity for VUR due to improper ureteral insertion and shortened IVT length resulting from ureteric induction defects.

VUR Does Not Resolve with Age in Frs2 $a^{\mathrm{ST}-/-}$ Mice

Although primary VUR spontaneously resolves in most patients, $\sim 33 \%$ have persistent reflux (16). To determine the natural history of VUR in Frs $2 \alpha^{S T-/-}$ mice, we performed cystograms in young adult (P30) mice (Figure 6). At P30, 14\% of control mice exhibit VUR, whereas $82 \%$ of $F r s 2 \alpha^{S T-/-}$ mice have reflux. (Table 2; $P<0.01)$. While every control had unilateral VUR, $22 \%$ of the mutants had bilateral VUR (Table 3). Lastly, while none of the controls had high-grade reflux, $18 \%$ of the mutants exhibited high-grade VUR (Table 3). These data illustrate that high rates of VUR in Frs $2 \alpha^{S T-/-}$ do not dissipate with age. Finally, while the Tbx18cre line is also likely deleting Frs $2 \alpha$ in ureter and bladder mesenchyme, H\&E stains of mutant ureters at P1 appear normal (Supplementary Figure S2) as did H\&E stains and anti-aSMA stains of mutant bladders at P30 (Supplementary Figure S3).

\section{DISCUSSION}

Previous studies have examined the role of Fgfr2 in ureteric induction defects and risks for reflux. Using a transgenic Pax3cre mouse line to delete Fgfr2 in both the peri-Wolffian duct ST and the metanephric mesenchyme, we observed ureteric induction defects and subsequent VUR as well as other anomalies such as duplex collecting systems and renal hypoplasia $(7,8)$. To resolve whether the effects were from the receptor actions in the peri-Wolffian duct ST or the metanephric mesenchyme, we previously deleted Fgfr2 with a Tbx18cre mouse line that drives cre expression in the periWolffian duct ST and not in the metanephric mesenchyme. Results from this study demonstrated that Fgfr2 signaling in the peri-Wolffian duct ST regulated the ureteric bud induction site, preventing VUR and other urinary tract anomalies (9). To elucidate which receptor signaling docking protein mediates the effects of Fgfr2 in the ST, we utilized Tbx 18 cre line to conditionally delete Frs $2 \alpha$.

Similar to the Tbx18cre; Fgfr $2^{\text {flfl }}$ line, Frs $2 \alpha^{\mathrm{ST}-/-}$ mice have ureteric bud induction defects, as evidenced by the extreme variability of mutant CND lengths (Wolffian duct segment between the ureteric bud base and the cloaca) when

Table 2. Incidence and severity of VUR in P1 control and Frs $2 a^{S T-/-}$ mice

\begin{tabular}{|c|c|c|c|c|c|c|}
\hline \multirow[t]{2}{*}{ Genotype } & \multicolumn{3}{|c|}{ Incidence of reflux } & \multirow{2}{*}{$\frac{\text { Reflux laterality }}{\text { Unilateral }}$} & \multicolumn{2}{|c|}{ Reflux grade } \\
\hline & Total & Male & Female & & I-II & III-IV \\
\hline Control & 4/58 (7\%) & $2 / 34(6 \%)$ & $2 / 24(8 \%)$ & 4/4 (100\%) & 4/4 (100\%) & 0/4 (0\%) \\
\hline
\end{tabular}



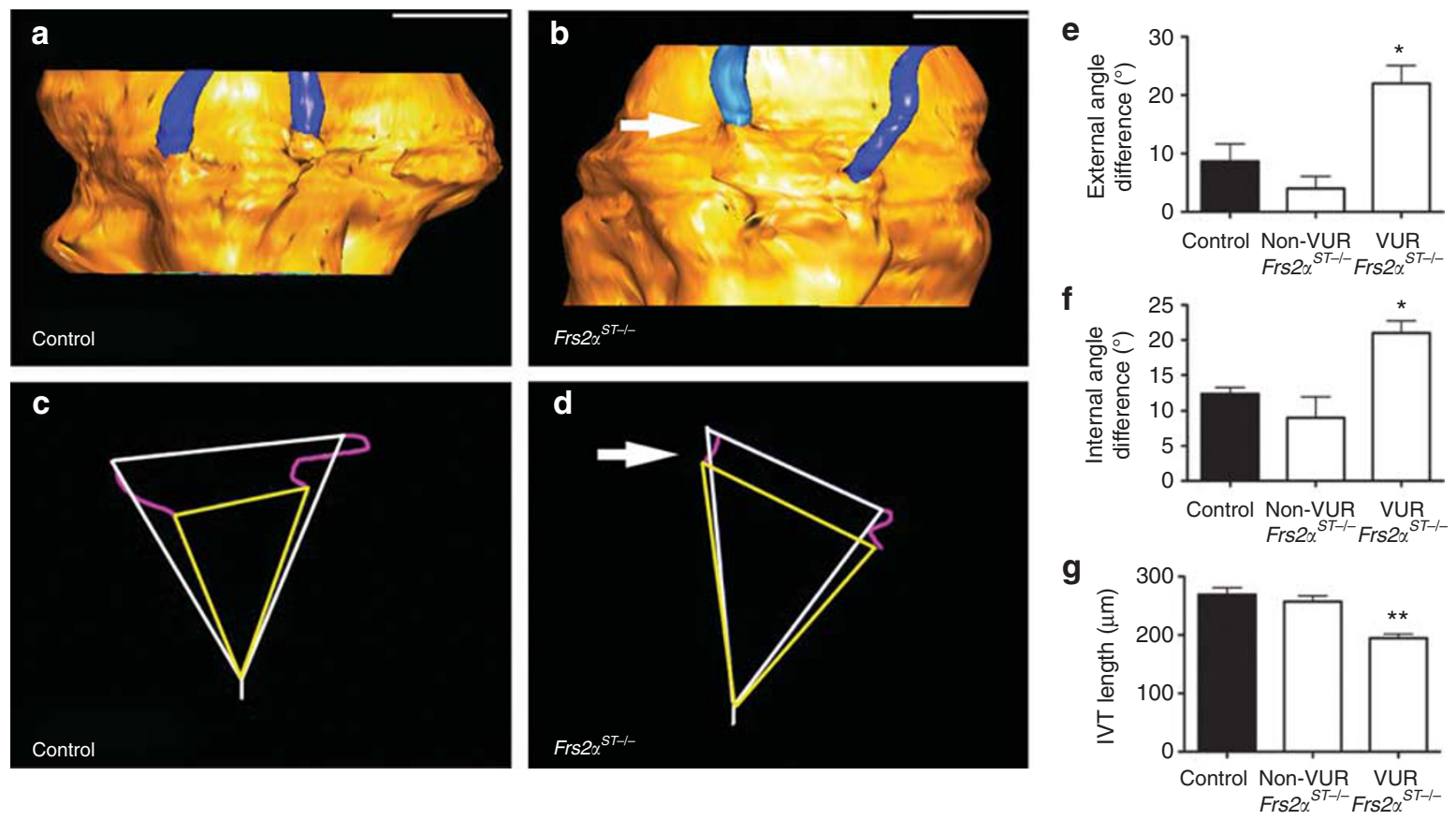

Figure 5. Refluxing ureters in $\mathrm{P} 1 \mathrm{Frs} 2 \mathrm{a}^{\mathrm{ST-/-}}$ mice have improper ureteral insertion and shortened intravesicular tunnel (IVT) lengths. (a,b) Representative 3D reconstruction reveal similar bladder insertion points of both control ureters (a, blue) into the bladder (yellow), but a high and lateral insertion of the right Frs2 $a^{S T-1-}$ ureter (arrow) into the bladder. (c,d) Triangles connecting the external ureter (white) and internal (yellow) insertion points and the bladder neck (from panels $\mathbf{a}$ and $\mathbf{b}$ ) illustrate similar insertion site angles in the control, indicating relatively parallel levels of insertion (c); however, the high and lateral refluxing Frs $2 a^{S T-/-}$ ureter (arrow) leads to triangles with very different insertion point angles (d). (e,f) Graphs confirm that compared to control and non-refluxing Frs $2 a^{S T-/-}$ mice, Frs $2 a^{S T-/-}$ mice with unilateral reflux have much larger differences in external (e) and internal (f) insertion site angles. (g) Graph revealing that mean IVT length (visualized as purple lines in panels $\mathbf{c}$ and $\mathbf{d}$ ) is significantly shorter in refluxing Frs $2 a^{S T-/-}$ ureters vs. control and non-refluxing Frs $2 a^{S T-/-}$ ureters $(n=3)$. Values = mean+SD. One-way analysis of variance ${ }^{*} P<0.05,{ }^{* *} P<0.01$. Bar $=500 \mu \mathrm{m}$.
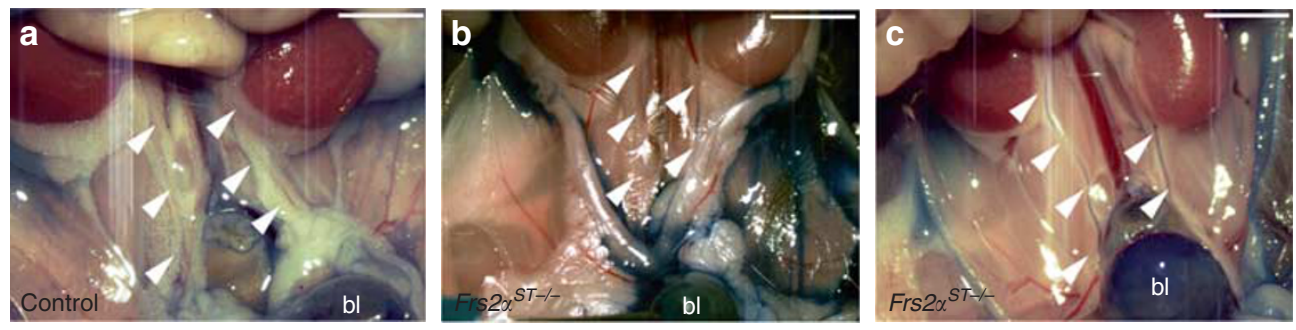

Figure 6. Cystograms in P30 Frs $2 a^{S T-/-}$ and control mice. (a) Representative control showing dye in the bladder (bl), but not in either ureter (arrowheads). (b,c) Representative Frs $2 a^{S T-/-}$ mice with left, unilateral (b) and bilateral (c) reflux from the bladders (bl) into the ureters (arrowheads). Bar $=5 \mathrm{~mm}$.

Table 3. Incidence and severity of VUR in P30 control and Frs $2 a^{S T-/-}$ mice

\begin{tabular}{|c|c|c|c|c|c|c|}
\hline \multirow[t]{2}{*}{ Genotype } & \multicolumn{3}{|c|}{ Incidence of reflux } & \multirow{2}{*}{$\frac{\text { Reflux laterality }}{\text { Unilateral }}$} & \multicolumn{2}{|c|}{ Reflux grade } \\
\hline & Total & Male & Female & & I-II & III-IV \\
\hline Control & $2 / 14(14 \%)$ & $2 / 7(29 \%)$ & 0/7 (0\%) & $2 / 2(100 \%)$ & $2 / 2(100 \%)$ & $0 / 2(0 \%)$ \\
\hline
\end{tabular}

* $P<0.01$ vs. control.

compared with that in the littermate controls. Apoptosis and proliferation were unaltered in $F r s 2 \alpha^{S T-/-}$ CNDs vs. control littermates suggesting that the differences between the genotypes are not because of persistence of or premature loss of the CND. Moreover, the previous Tbx18cre; Fgfr $2^{f l f l}$ study suggested that the molecular mechanism for ureteric induction defects is due to the decreased Bmp4 expression and signaling in the peri-Wolffian duct ST adjacent, as has been 


\section{Articles | Narla et al.}

shown in Bmp4 heterozygous mice (9). Accordingly, we found an $\sim 40 \%$ reduction in Bmp4 mRNA and pSmad 1/5/8 protein expression in Frs $2 \alpha^{S T-/-}$ urogenital ridges when compared with controls. While a one allele loss of Bmp4 has previously been shown to cause ureteric induction defects, these data are the first to suggest that this loss leads to a defect in canonical Bmp4 signaling (5). Taken together, these data strongly suggest that peri-Wolffian signaling through Fgfr2 is mediated mostly by Frs $2 \alpha$, which in turn drives expression and canonical signaling of Bmp4 to constrain the ureteric bud induction site.

The Frs $2 \alpha^{S T-/-}$ ureteric bud induction defects led to aberrant ureter insertion into the bladder and high rates of VUR, as would be predicted by Drs Mackie and Stephens' studies in humans with VUR $(4,17)$. 3D reconstructions revealed that refluxing P1 mutant ureters inserted either high and lateral or low and medial into the bladder compared with the non-reflexing ureter. In addition, mean refluxing Frs $2 \alpha^{S T-1-}$ intravesicular lengths were shortened in comparison with non-refluxing ureters, which is also a known risk for reflux in humans (4). On the basis of the data from Tbx18cre; $F g f r 2^{f l / f l}$ and Tbx18cre; Frs $2 \alpha^{f l / f l}$ mice, the threshold intravesicular length for reflux appears to be $\sim 200 \mu \mathrm{m}$ or less (9). While Frs $2 \alpha$ is also likely deleted in postnatal ureter and bladder mesenchymal derivatives (muscle and lamina propria), these structures appear normal in mutants (Supplementary Figures S2 and S3) and are not likely to be reasons for VUR in the mutants. While we cannot exclude that the mutant ureters have peristalsis defects (which could be contributing to reflux in live mice), our cystogram assays are on euthanized mouse urinary tracts, none of which are undergoing peristalsis.

Notably, both control and Frs $2 \alpha^{S T-/-}$ mice exhibited reflux during analysis; however, the reflux observed in control mice was similar to that observed in P1 controls of other recent studies indicating a similar degree of VUR susceptibility in our controls as compared with previously published findings $(8,9)$. In comparing the current study to our previous studies, we observed some differences in P1 reflux rates between Fgfr $2^{S T-/-}$ mice and Frs $2 \alpha^{S T-/-}$ mice (9). While control mice in both studies exhibit comparable rates of reflux, $~ 75 \%$ of P1 Fgfr $2^{S T-1-}$ mice had reflux when compared with $~ 50 \%$ in $\operatorname{Frs} 2 \alpha^{S T-1-}$ mice in this study (9). One explanation for the differences is that downstream docking proteins outside of Frs2 $\alpha$ (such as Plcw or Crk2) may have minor roles in mediating effects of Fgfr2 signaling in peri-Wolffian duct ST. Other explanations include operator differences between the studies or some drift in the background strain between the Fgfr2 mutants in the previous study and the Frs $2 \alpha$ mutants in the present study. Other studies from our laboratory using Six 2 cre lines to manipulate Fgfr and/or Frs $2 \alpha$ expression in nephron progenitors show a slight rescue of nephron progenitor depletion in the latter mutants vs. the former (12). In contrast, in the ureteric epithelium, Fgfr2 and Frs $2 \alpha$ appear to act independently to regulate ureteric morphogenesis (13). Taken with the current study, Frs $2 \alpha$ does appear to be the major docking protein for Fgfrs in metanephric and peri-Wolffian duct mesenchyme, but not in ureteric epithelium. It is not clear whether this pattern extends to other developing mesenchymal and epithelial tissues.

Finally, unlike our previous studies, we examined the rates of VUR in Frs $2 \alpha^{S T-/-}$ mice with age. Interestingly, we noted persistent, if not higher, rates of VUR in mutants aged to P30. One explanation may be that we used different needle sizes for the P1 vs. P30 (30 gauge vs. 25 gauge, respectively); we and other investigators performing cystograms in mice have found that 25 gauge needles fill the P1 bladders too quickly and generate very high pressures, whereas, 30 gauge needles are too small to adequately fill the P30 bladders in a timely manner $(18,19)$. In support of the "needle" hypothesis is that the P30 control rates of reflux appear to be higher than those at P1. Another potential explanation for the persistence/ worsening of reflux in P30 mutants may be the effects of Tbx18cre-driven loss of Frs $2 \alpha$ in bladder mesenchyme, which are yet to be determined. Thus the apparently higher rates of reflux in P30 Frs $2 \alpha^{S T-/-}$ mice may be secondary to bladder defects. Given that the Frs $2 \alpha^{S T-/-}$ mice have progressive limb defects, we have been unable to perform many assays to interrogate bladder function (such as void stain on paper). Regardless of the cause, the apparently persistent reflux in the Frs $2 \alpha$ mutants raises interesting clinical questions, including whether perturbations/mutations in the Fgfr/Frs $2 \alpha$ family may be linked to patients that have persistent reflux.

\section{SUPPLEMENTARY MATERIAL}

Supplementary material is linked to the online version of the paper at http://www.nature.com/pr

\section{STATEMENT OF FINANCIAL SUPPORT}

The study was supported by NIH grants R01 DK104374 (C.M.B.) and P30 DK079307 (Tom Kleyman, PD; CMB co-I).

Disclosure: The authors declare no conflict of interest.

\section{REFERENCES}

1. Murawski IJ, Gupta IR. Gene discovery and vesicoureteric reflux. Pediatr Nephrol 2008;23:1021-7.

2. Fillion ML, Watt CL, Gupta IR. Vesicoureteric reflux and reflux nephropathy: from mouse models to childhood disease. Pediatr Nephrol 2014;29:757-66.

3. Dressler GR. The cellular basis of kidney development. Annu Rev Cell Dev Biol 2006;22:509-29.

4. Mackie GG, Awang H, Stephens FD. The ureteric orifice: the embryologic key to radiologic status of duplex kidneys. J Pediatr Surg 1975;10:473-81.

5. Miyazaki Y, Oshima K, Fogo A, Hogan BL, Ichikawa I. Bone morphogenetic protein 4 regulates the budding site and elongation of the mouse ureter. J Clin Invest 2000;105:863-73.

6. Poladia DP, Kish K, Kutay B, et al. Role of fibroblast growth factor receptors 1 and 2 in the metanephric mesenchyme. Dev Biol 2006;291: 325-39.

7. Hains D, Sims-Lucas S, Kish K, Saha M, McHugh K, Bates CM. Role of fibroblast growth factor receptor 2 in kidney mesenchyme. Pediatr Res 2008;64:592-8.

8. Hains DS, Sims-Lucas S, Carpenter A, et al. High incidence of vesicoureteral reflux in mice with Fgfr2 deletion in kidney mesenchyma. J Urol 2010;183:2077-84. 
9. Walker KA, Sims-Lucas S, Di Giovanni VE, et al. Deletion of fibroblast growth factor receptor 2 from the peri-wolffian duct stroma leads to ureteric induction abnormalities and vesicoureteral reflux. PLoS ONE 2013;8:e56062.

10. Sims-Lucas S, Di Giovanni V, Schaefer C, Cusack B, Eswarakumar VP, Bates CM. Ureteric morphogenesis requires Fgfr1 and Fgfr2/Frs2alpha signaling in the metanephric mesenchyme. J Am Soc Nephrol 2012;23: 607-17.

11. Ong SH, Guy GR, Hadari YR, et al. FRS2 proteins recruit intracellular signaling pathways by binding to diverse targets on fibroblast growth factor and nerve growth factor receptors. Mol Cell Biol 2000;20:979-89.

12. Di Giovanni V, Walker KA, Bushnell D, et al. Fibroblast growth factor receptor-Frs2alpha signaling is critical for nephron progenitors. Dev Biol 2015;400:82-93.

13. Sims-Lucas S, Cusack B, Eswarakumar VP, Zhang J, Wang F, Bates CM. Independent roles of Fgfr2 and Frs2alpha in ureteric epithelium. 2011Development 138:1275-80.
14. Wang Y, Tripathi P, Guo Q, Coussens M, Ma L, Chen F. Cre/lox recombination in the lower urinary tract. Genesis 2009;47:409-13.

15. Lebowitz RL, Olbing $\mathrm{H}$, Parkkulainen KV, Smellie JM, Tamminen-Mobius TE. International system of radiographic grading of vesicoureteric reflux. International Reflux Study in Children. Pediatr radiol 1985;15:105-9.

16. Peters CA, Skoog SJ, Arant BS Jr, et al. Summary of the AUA guideline on management of primary vesicoureteral reflux in children. J Urol 2010;184: 1134-44.

17. Mackie GG, Stephens FD. Duplex kidneys: a correlation of renal dysplasia with position of the ureteral orifice. J Urol 1975;114:274-80.

18. Boualia SK, Gaitan Y, Murawski I, Nadon R, Gupta IR, Bouchard M. Vesicoureteral reflux and other urinary tract malformations in mice compound heterozygous for Pax2 and Emx2. PLoS ONE 2011;6:e21529.

19. Murawski IJ, Watt CL, Gupta IR. Vesico-ureteric reflux: using mouse models to understand a common congenital urinary tract defect. Pediatr Nephrol 2011;26:1513-22. 